\title{
A Method for Measuring the Upper Limb Motion and Computing a Compatible Exoskeleton Trajectory
}

\author{
N. Jarrassé, V. Crocher, G. Morel \\ Pierre et Marie Curie University - Institut des Systèmes Intelligents et de Robotique - CNRS - UMR 7222 \\ 4 place Jussieu, 75005 Paris - France \\ Emails : \{jarrasse, crocher, morel $\} @$ isir.upmc.fr
}

\begin{abstract}
This paper deals with the problem of computing trajectories for an exoskeleton that match a motion recorded on a given subject. Literature suggests that this problem can be solved by reconstructing the subject's joint motion using one of the numerous models available, and then feeding the exoskeleton with the joint trajectories. This is founded on the assumption that the exoskeleton kinematics reproduces the human kinematics. In practice, though, mismatches are unavoidable and lead to inaccuracies.

We thus developed a method that is primarily based on an appropriate mechanical design: passive mechanisms are used to connect the exoskeleton with splints wore by the subject, in such a way that, within the workspace, there always exists a posture of the exoskeleton compatible with a given position and orientation of the splints. The trajectory computing method, by itself, consists of recording the position and orientation of the splints thanks to a conventional 3D motion tracker and to exploit standard robotics tools in order to compute an exoskeleton posture compatible with the measured human posture. Conclusive experimental results involving an existing 4 DoF upper-limb exoskeleton are shown.
\end{abstract}

\section{INTRODUCTION}

Whatever the particular use they are designed for augmenting human force capabilities [1], helping a patient during neurophysical rehabilitation [2] or simply providing force feedback for haptics or telemanipulation [3] - the major purpose of exoskeletons is to move in synergy with a human subject while transmitting forces distributed along its limb. Possible modes of operation range from so-called passive mode, where the subject passively follows the motion imposed by the robot, to active mode, where the robot shall follow the motion intended by the subject without resisting. In most cases [4], [5], [6], the control law requires a reference trajectory for the exoskeleton. This reference trajectory shall be designed in such a way that it corresponds to a given trajectory for the human limb. The following question is thus central in exploiting exoskeletons for assisting human motion: given an individual subject realizing a movement with his/her limb, how to compute a trajectory for the exoskeleton that is kinematically compatible with this movement? Because the main idea in designing an exoskeleton is to reproduce the human joint kinematics, the dominant paradigm to answer this question is to reconstruct a joint motion for the subject's limb, and to directly feed the robot with this joint motion.
To this aim, a first concern is the tracking of the limb's motion and its representation in an appropriate joint space. The literature on this issue is rather dense in the biomechanics community [7]. Usually, a model of rigid bodies (the bones) connected through joints is used. Across the literature, models vary in terms of the number of Degrees of Freedom (DoFs) considered for each joint, and the nature of the joints. For example, the upper limb is modelled as a 7 DoF system in [8] with conventional joints (pivots), whereas in [9], joints are modeled by bone surfaces sliding on each other, said surfaces being identified from 3D MRI images. Moreover, methods for identifying the joint motion, i.e. for matching a chosen rigid-jointed model with a set of recorded data, suffer from the fact that the bones motion cannot be recorded. Rather, landmarks must be placed on the subjects skin and therefore move differently from the bones. All in one, there is no consensus today, across the literature, on the question of joint motion recording and its reconstruction. Considering now the robotics point of view, using the jointby-joint mapping paradigm for programming the trajectory of an exoskeleton supposes that the device kinematics is identical, or at least very similar, to the subject's limb kinematics. In the exoskeleton design literature, this challenge is addressed by minimizing the kinematic differences between the two mechanical chains. For example, lockable sliders have been proposed to adjust robot limbs length to the subject's ones, [10], [11]. Alternatively, extra degrees of freedom can be added to the robot structure to increase its capacity of adaptation, [12]. A major drawback of these approaches is that the exact kinematic correspondence seems to be impossible to reach because of the complexity of the human limb joints. Therefore, when connecting the two chains through mechanical fixations, the system becomes statically undetermined, or hyperstatic, which means that uncontrollable forces may appear at the interaction points. Consequently, the use of a simple one-to-one mapping to feed the robot with recorded human joint trajectories will lead to poor results if no precise (and thus complex) models of the human limb and robot kinematics are built to compensate for differences and offsets between joints.

Based on this analysis, we developed in [13] a methodology for removing hyperstaticity by adding passive DoF between the robot and the splints attached to the subject's limbs. This method, applied to a 4 DoF device in section II, allows 
to connect a given exoskeleton to a given subject while formally guaranteeing isostaticity, i.e. full force controllability, even when the kinematics of the two chains differ. This property opens an elegant and simple solution to the trajectory computation problem considered in the present paper. Indeed, kinematic compatibility is a property that is dual to isostaticity [14]. In other words, for any position and orientation of the splints, there exists a corresponding joint position for the set constituted by the exoskeleton and the passive fixation mechanisms.

Therefore, the problem of reproducing a human limb posture thanks to an exoskeleton can be simply solved: firstly, the position and orientation of the splints wore by the subject during unassisted motions are recorded; secondly, an inverse kinematics model is computed for the exoskeleton and the passive fixations; thirdly, the joint position of the exoskeleton is extracted from the previous result. As a result, no kinematic model of the human limb is required, only the robot kinematic model is used.

The section III-A details the kinematic compatibility problem while the section III-B explains the general method and section III-C its application to ABLE exoskeleton. Experimental results are given in section IV.

\section{AVOIDING HYPERSTATICITY USING PASSIVE MECHANISMS ON ABLE EXOSKELETON}

The method presented in [13] is applied to a 4 axis arm exoskeleton with the aim of designing some passive DoF fixation mechanism to avoid the appearance of uncontrollable forces at the interface and, dually, to ensure that a robot posture exists for any recorded position and orientation of the subject arm, and that without building any complex model of the human kinematic.

\section{A. Application of the methodology}

ABLE (see Fig. 1) is a 4 axis exoskeleton that has been designed by CEA-LIST [15] on the basis of an innovative screw-and-cable actuation technology ([16]). Its kinematics

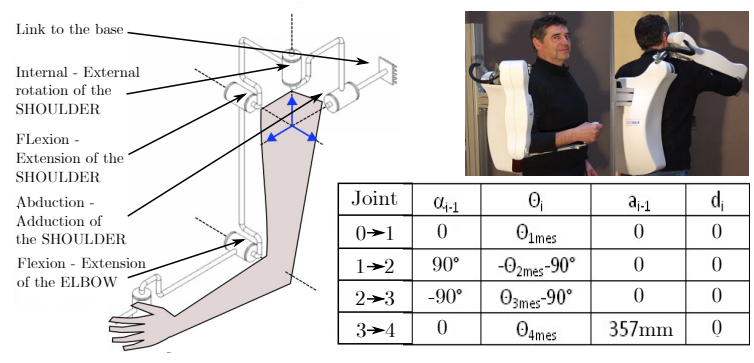

Fig. 1. Kinematics of ABLE

is composed of a shoulder spherical joint composed of 3 coincident pivots and a 1 DoF pivot elbow. The forearm, terminated by a handle, is not actuated. Details on this robot can be found in [15].

Applying method from [13] to this exoskeleton and its two fixations lead to the representation shown in Fig. 2. Considering that the robot and the limbs are connected

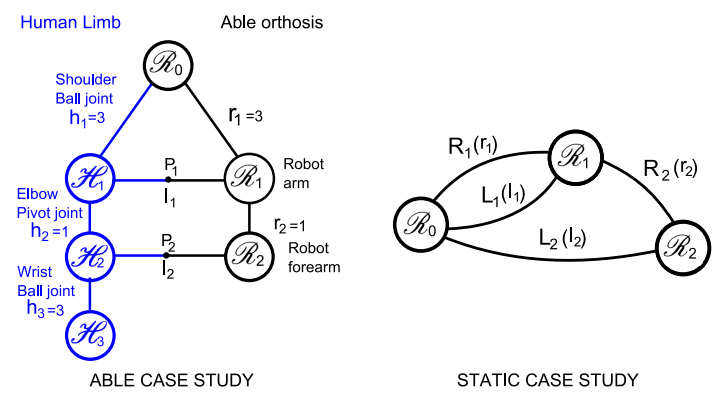

Fig. 2. Schematic of the ABLE and human arm coupling

through $n$ fixations connecting each human limb segment to a robot one and that each fixation is a mechanism consisting in a passive kinematic chain which connects a human body to a robot body.

Then the total number of passive DoF to be added is given by the following set of equations:

$$
\begin{gathered}
\forall i \in 1 \cdots n, \sum_{j=1}^{i}\left(l_{j}+r_{j}\right) \geq 6 . i \\
\forall i \in 1 \cdots n, \sum_{j=1}^{i-1}\left(l_{j}+r_{j}\right)+r_{i} \leq 6 . i \\
\sum_{j=1}^{n}\left(l_{j}+r_{j}\right)=6 . n
\end{gathered}
$$

with $l_{i}$ the connectivity of the fixation mechanism $i$ (fixation can be an embedment - $l_{i}=0$ - or can release several DoFs, such that: $\left.\forall i \in\{1, . ., n\}, \quad 0 \leq l_{i} \leq 5\right)$, and with $r_{i}$ the connectivity of each robot active joint.

Using this set of equations on the ABLE structure described on Fig. 2 leads to:

$$
\sum_{j=1}^{n=2} l_{j}=12-\sum_{j=1}^{n=2} r_{j}=12-(3+1) \quad \Rightarrow l_{1}+l_{2}=8
$$

Moreover, for the first fixation, Eq. (1a) and (1b) give:

$$
6-r_{1} \leq l_{1} \leq 6 \quad \Rightarrow \quad 3 \leq l_{1} \leq 5 .
$$

Since the total number of DoFs is fixed, the tree of possible solutions consists here of three parallel branches where $l_{1}$ is chosen between 3 and 5 and $l_{2}=8-l_{1}$ (i.e. three possible couple of solution). As it is explained in [13], we choose the solution with $4 \mathrm{DoF}$ freed at each fixation, especially because it reproduces the method used by physical therapists to assist patients in generating internal rotations of the shoulder without torsion to the tissue, through the application of two opposite forces on the elbow and on the hand of the patient.

\section{B. Fixations realization}

The two fixations mechanisms are identical. They shall generate three independent rotations and one translation along the limb. The mechanism used to realize this function consists of three successive pivot joints which axis coincide 


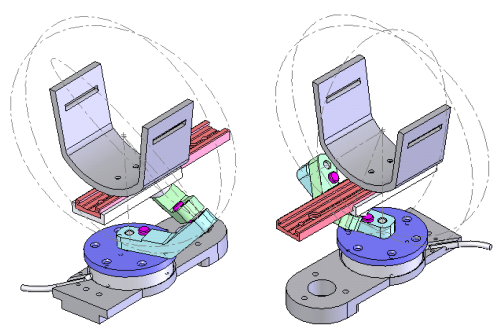

Fig. 3. CAD view of the fixation's mechanism (rear and front)

and one slider whose axis is parallel to human limb (see Fig $3)$.

The fixations were dimensioned differently: one to allow forearm pronosupination and the other not to collide with arm tissues. As a result, possible motions left by the passive fixations have the ranges defined in Table I. These fixations

\begin{tabular}{|l|c|c|}
\hline DoF & Arm Fixation & Forearm Fixation \\
\hline Rotation1 $(\perp$ to the limb axis) & $360^{\circ}$ & $360^{\circ}$ \\
\hline Rotation2 $(\perp$ to the limb axis) & $90^{\circ}$ & $90^{\circ}$ \\
\hline Rotation3 (around the limb axis) & $110^{\circ}$ & $110^{\circ}$ \\
\hline Translation & $100 \mathrm{~mm}$ & $100 \mathrm{~mm}$ \\
\hline
\end{tabular}

TABLE I

are mounted on ABLE exoskeleton. Arm fixation is placed near the elbow, just under the triceps whereas forearm fixation is placed near the wrist.
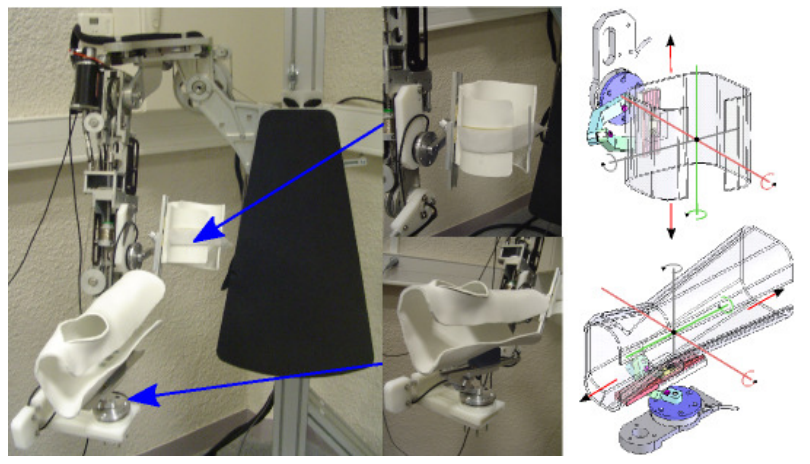

Fig. 4. ABLE with the two splints, the two passive mechanisms and the two F/T sensors (at exoskeleton/passive mechanisms interaction points).

Thermoformable materials were also used to create two splints perfectly adapted to human morphology. These splints are serially connected to the last fixation body. Wrist splint was specifically created to lock the wrist flexions which are not studied here. Only passive pronosupination is allowed.

\section{Method to compute a robot posture COMPATIBLE WITH AN HUMAN ONE}

\section{A. Kinematic compatibility}

Since the design method presented in the previous section guarantees global aisostaticity, it, dually, satisfies kinematic compatibility. Given a human arm posture defined by its joint position $\mathbf{q}_{h} \in \mathbb{R}^{n_{h}}$, the question to be solved here is how to compute a robot posture $\mathbf{q}_{r} \in \mathbb{R}^{n_{r}}$ that is kinematically compatible. In the general case, the kinematics of the robot and those of the human limb differ, in such a way that $\mathbf{q}_{r} \neq \mathbf{q}_{h}$. In fact, even their dimension differ for most of the existing exoskeletons $\left(n_{r} \neq n_{h}\right)$.

With the proposed design method, one can define for the robot an augmented joint vector:

$$
\mathbf{q}_{t}^{\mathrm{T}}=\left[\mathbf{q}_{r}^{\mathrm{T}} \mathbf{q}_{l}^{\mathrm{T}}\right]^{\mathrm{T}}
$$

where $\mathbf{q}_{l} \in \mathbb{R}^{n_{l}}$ is the joint position of the passive mechanisms used to connect the robot to the splints installed on the subject, with $n_{l}=\sum_{j=1}^{n} l_{j}$.

From Eq. 1c, one can see that:

$$
\operatorname{dim}\left(\mathbf{q}_{r}\right)+\operatorname{dim}\left(\mathbf{q}_{l}\right)=6 n,
$$

Furthermore, the method also guarantees a full kinematic rank, meaning that, if the translational and rotational velocities of the $n$ splints with respect to the base body are grouped into a $6 n$-dimensional vector $\dot{\mathbf{x}}$, the mapping

$$
\dot{\mathbf{X}}=J\left(\mathbf{q}_{t}\right) \dot{\mathbf{q}}_{t}
$$

is non singular.

Therefore, as $\dot{\mathbf{X}}$ lies in the range space of the Jacobian, computing $\mathbf{q}_{r}$ compatible with any human arm posture pertains to standard inverse kinematics of a $6 n$ joint robot.

\section{B. Posture computation}

To compute a compatible robot configuration for a given human arm posture, a two-step procedure was used.

1) Splints motions recording: A motion capture device (a Polaris ${ }^{\circledR}$ system from Northern Digital Inc.) was used for recording positions and orientations of the human limb segments that will be controlled afterwards by the robot. In order to simplify the calculation of these trajectories in the robot frame $\mathbf{R}_{r}$, Polaris ${ }^{\circledR}$ targets with their optical markers were placed on the rigid splints that are used to fix the human segment to the last body of the passive fixation mechanism.

Subject wearing the splints is asked to perform 3D movements that are recorded, and, thanks to a preliminary identification of the postures of the splint center in their respective splint frames $\mathbf{R}_{s_{i}}$ (with $i \in[1 \cdots n]$ ), position and orientation trajectories of these $i$ points in the robot frame $\mathbf{R}_{r}$ are calculated: this provides $\mathbf{X}_{i}(t)$.

2) Computing $\mathbf{q}_{t}(t)$ : In order to obtain $\mathbf{q}_{t}(t)$ corresponding to $\mathbf{X}(t)$, a standard approach was used to compute $\mathbf{q}_{t}(t)$ from the equation

$$
\dot{\mathbf{q}}_{t}=J^{\dagger}\left(\mathbf{q}_{t}\right) \dot{\mathbf{X}}
$$


with $J^{\dagger}\left(\mathbf{q}_{t}\right)$ the pseudoinverse of $J$, also called the MoorePenrose inverse of $J . J$ is defined as

$$
\dot{\mathbf{q}}_{t}=\left[\begin{array}{lll}
J_{1}^{\dagger} & J_{2}^{\dagger} \ldots J_{n}^{\dagger}
\end{array}\right] \cdot\left(\begin{array}{c}
\dot{\mathbf{X}}_{1} \\
\dot{\mathbf{X}}_{2} \\
\ldots \\
\dot{\mathbf{X}}_{n}
\end{array}\right)
$$

with $\dot{\mathbf{X}}_{i}$ the posture in the robot frame $\mathbf{R}_{r b_{i}}$ of the $i^{\text {th }}$ robot body. The pseudoinverse allows to compute a 'best guess' (in the sense of least squares) solution to this system of equations.

Finally in order to obtain robot joint trajectories, $\mathbf{q}_{r}$ is simply extracted from $\mathbf{q}_{t}$ with $\mathbf{q}_{r}=\left[\begin{array}{ll}\mathbf{I}_{n_{r}} & \mathbf{0}_{n_{l}}\end{array}\right] \mathbf{q}_{t}$

\section{Application to the ABLE exoskeleton}

The 4 DoF exoskeleton ABLE with its passive mechanisms has two interaction points (one for the arm, one for the forearm) and so 12 DoF including passive ones. The two splints, used to connect the human limb, are shown on Fig. 4 with their Polaris ${ }^{\circledR}$ targets.

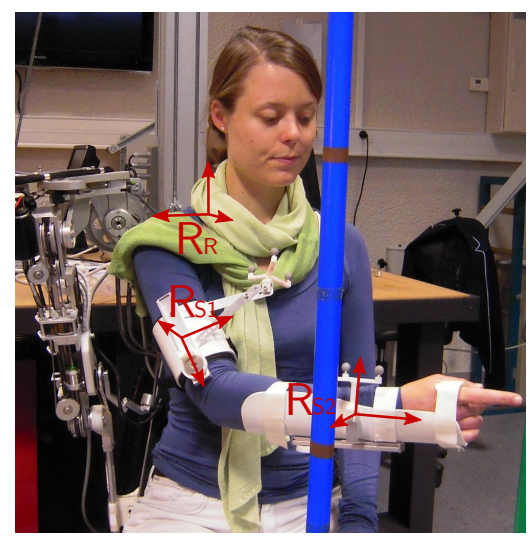

Fig. 5. A subject, wearing the two splints, with Polaris ${ }^{\circledR}$ targets and the used frames $\mathbf{R}_{r}, \mathbf{R}_{s_{1}}$ and $\mathbf{R}_{s_{2}}$.

A preliminary identification of the fixation ball joint centers position $P_{1}$ and $P_{2}$ in each robot segment frame $\mathbf{R}_{r b_{1}}$ and $\mathbf{R}_{r b_{2}}$ has been made together with an identification of the position and orientation of the Polaris ${ }^{\circledR}$ targets frames $\mathbf{R}_{t_{1}}$ and $\mathbf{R}_{t_{2}}$ in the splint frames $\mathbf{R}_{s_{1}}$ and $\mathbf{R}_{s_{2}}$. This frames are so located respectively at the center of the arm and the forearm of the subject. This allows to obtain, after the recording of subject wearing only the splints, the trajectories of the arm and forearm axis in the robot frame $\mathbf{R}_{r}$.

For a given limb posture, $\mathbf{R}_{t_{1}}$ and $\mathbf{R}_{t_{2}}$ are recorded by Polaris ${ }^{\circledR}$ system in the robot frame $\mathbf{R}_{r}$. Thanks to the identification, the splint frames $\mathbf{R}_{s_{1}}$ and $\mathbf{R}_{s_{2}}$, corresponding to $\mathbf{X}$ can be obtained. Robot compatible configuration $\mathbf{q}_{t}$ is then computed as explained in III-B.2. From this 12 elements configuration, only the 4 interesting ones (i.e. the 4 actuated $\mathrm{DoF})$ are extracted for a subsequent use.

\section{EXPERIMENTAL RESULTS}

In order to validate the proposed method, several experiments have been conducted.

\section{A. Experimental setup}
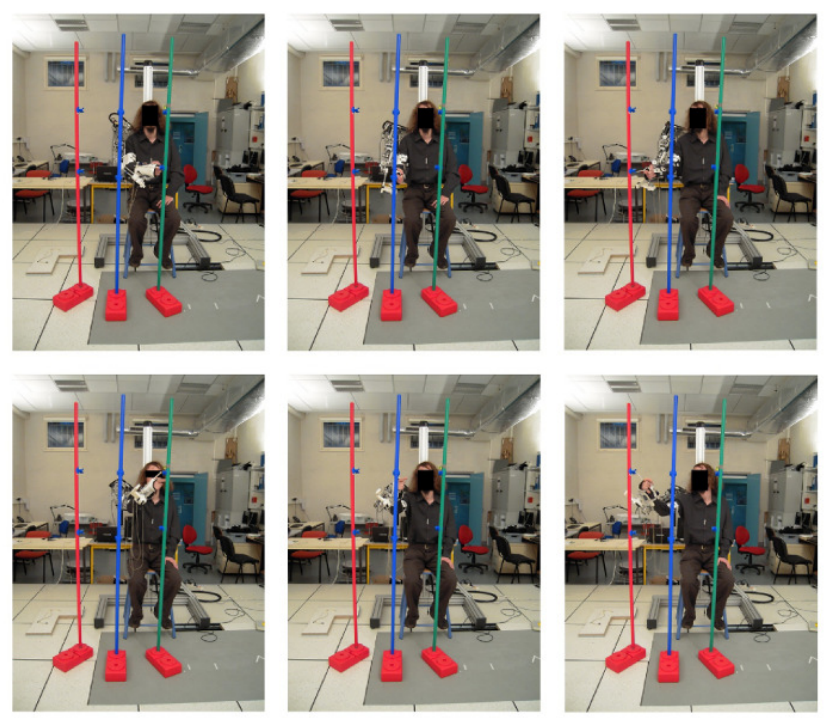

Fig. 6. A subject during the experiment making the 6 different postures

In a first step, four naive healthy subjects wearing splints fitted with optical markers and seated against exoskeleton back (but not connected to it) are asked to reach 6 different points in his/her workspace. The points are materialized by points on rods (see Fig. 6). These 6 points correspond to 6 different upper-limb postures, presented on Fig. 6.

Splints optical markers positions are recorded for each posture. Then, the previously defined method is applied to the recorded data in order to generate kinematically compatible robot joint configurations.

The subject is then connected to the exoskeleton through the two passive fixations and asked to let his/her arm be passively moved by the robot. Next, the exoskeleton, thanks to a position control loop, reaches the different recorded configurations with the subject inside. For each position, optical markers are once again recorded in order to allow a comparison of postures in the two conditions ("actively" reached without robot and "passively" reached with robot).

In a second step, with a single subject, a complete movement trajectory of the arm is recorded without the robot. Then, same method is used to reproduce the trajectory in the passive mode, by simply considering the trajectory as a succession of postures. The robot is then connected and used to make the subject's arm to reproduce the obtained trajectory, thanks to the position control loop. Optical markers positions are still recorded during the movement reproduction.

\section{B. Results}

In order to compare the postures in the two conditions, two measures are computed between each frame $\left(\mathbf{R}_{s_{1}}, \mathbf{R}_{s_{2}}\right)$ : the distance between the origin of the frames : $d_{1}$ and $d_{2}$, and the angle between the frames axis : $\theta_{1}$ and $\theta_{2}$, respectively for frames $\mathbf{R}_{s_{1}}$ and $\mathbf{R}_{s_{2}}$. 
$d_{1}$ and $d_{2}$ are computed as the 3D distances between the frames origin points in the two conditions with and without robot :

$d_{1}=\sqrt{\left(x_{s 1 w /}-x_{s 1 w / o}\right)^{2}+\left(y_{s 1 w /}-y_{s 1 w / o}\right)^{2}+\left(z_{s 1 w /}-z_{s 1 w / o}\right)^{2}}$

$d_{2}=\sqrt{\left(x_{s 2 w /}-x_{s 2 w / o}\right)^{2}+\left(y_{s 2 w /}-y_{s 2 w / o}\right)^{2}+\left(z_{s 2 w /}-z_{s 2 w / o}\right)^{2}}$,

where $\left(x_{s 1 w /} ; y_{s 1 w /} ; z_{s 1 w /}\right)$ and $\left(x_{s 1 w / o} ; y_{s 1 w / o} ; z_{s 1 w / o}\right)$ are respectively the coordinates of the frame center, in the with and without robot conditions.

$\theta_{1}$ and $\theta_{2}$ are computed as follows :

$$
\begin{array}{r}
\theta_{1}=\operatorname{acos}\left(\frac{\operatorname{tr}\left(\mathbf{R} \operatorname{rot}_{s 1 w /} \mathbf{R} r o t_{s 1 w / o}-1\right)-1}{2}\right) \\
\theta_{2}=\operatorname{acos}\left(\frac{\operatorname{tr}\left(\mathbf{R} r o t_{s 2 w /} \mathbf{R} r_{s 2 w / o}{ }^{-1}\right)-1}{2}\right),
\end{array}
$$

where $\mathbf{R r o t}_{s 1 w /}$ and $\mathbf{R}$ rot $_{s 1 w / o}$ are the rotation matrixes extracted from $\mathbf{R}_{s_{1}}$ in the two conditions with and without robot for the arm and $\mathbf{R} r_{s 2 w /}$ and $\mathbf{R} r_{s 2 w / o}$ the rotation matrixes in the two conditions for the forearm.

These measures are presented on table II for each posture (averaged for the 4 subjects and standard deviation), and for the whole trial (mean on the 6 targets).

\begin{tabular}{|c|c|c|c|c|}
\hline Posture & $d_{1}(\mathrm{~mm})$ & $d_{2}(\mathrm{~mm})$ & $\theta_{1}(\mathrm{deg})$ & $\theta_{2}(\mathrm{deg})$ \\
\hline 1 & $13 \pm 7$ & $9 \pm 3$ & $9.8 \pm 7.6$ & $3.3 \pm 1.6$ \\
\hline 2 & $8 \pm 3$ & $9 \pm 3$ & $13.6 \pm 9.4$ & $8.8 \pm 6.5$ \\
\hline 3 & $10 \pm 4$ & $9 \pm 2$ & $10.9 \pm 10.5$ & $8.7 \pm 6.9$ \\
\hline 4 & $9 \pm 6$ & $12 \pm 5$ & $18.4 \pm 9.5$ & $9.5 \pm 1.7$ \\
\hline 5 & $13 \pm 5$ & $9 \pm 3$ & $10.8 \pm 3.9$ & $10.9 \pm 4.0$ \\
\hline 6 & $10 \pm 5$ & $12 \pm 4$ & $14.2 \pm 7.7$ & $5.9 \pm 4.2$ \\
\hline \hline Mean & $11 \pm 3$ & $10 \pm 2$ & $13.0 \pm 7.1$ & $7.8 \pm 1.5$ \\
\hline
\end{tabular}

TABLE II

RESULTS FOR EACH POSTURE.

For illustration purposes, both positions and orientations of the frames of the arm and the forearm are presented on Fig. 7. All the six postures are presented, for subject \#1, in the two conditions.

The results shows the efficiency of proposed method to calculate a compatible posture for the exoskeleton with an error of $11 \mathrm{~mm}$ and $10 \mathrm{~mm}$ respectively at the two interaction points. Moreover the error angles are $13.0^{\circ}$ and $7.8^{\circ}$ for the two points between the original postures and the calculated ones. Considering the exoskeleton flexibility and the splints identification error $(4.7 \mathrm{~mm})$, we could consider that postures could be calculated with a good confidence. Moreover, a part of the error remaining could be explained by the possible movements of the subject pronosupination, which is not controlled by the exoskeleton and not locked by the forearm splint, as explained in section II-B. Indeed, depending on the subject morphology, the center of the forearm splint does not exactly coincide with the subject

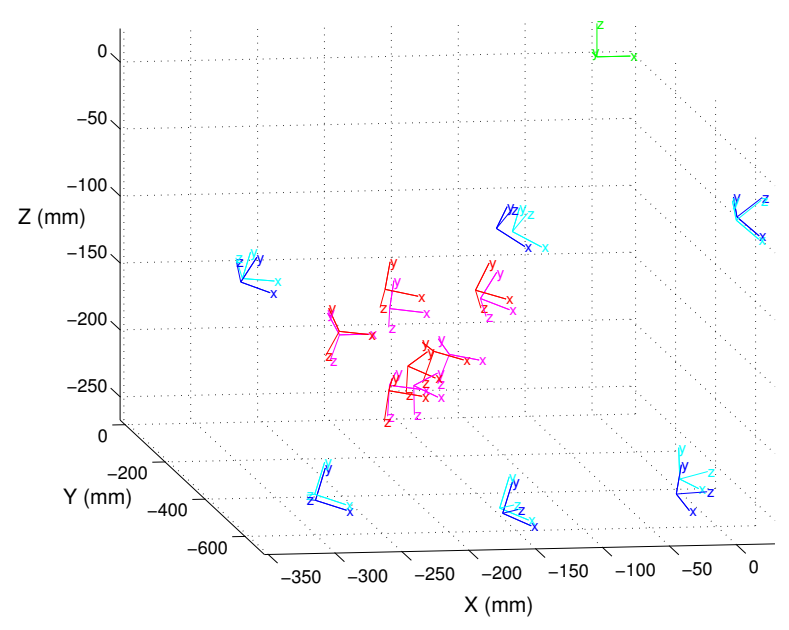

Fig. 7. Frames of the arm $\left(\mathbf{R}_{s 1}\right)$ without (red) and with robot (magenta). Frames of the forearm $\left(\mathbf{R}_{s 2}\right)$ without (blue) and with robot (cyan). The green frame stand for the global frame origin $\mathbf{R}_{r}$.

pronosupination axis.

For the second part of the experiments a trajectory is recorded and reproduced by the exoskeleton. The recorded successive positions of the arm and forearm frames are printed on Fig. 8 in the two conditions (with and without the robot).

As for the previous results (postures), for a trajectory, errors are about $10-15 \mathrm{~mm}$. This result essentially shows that a generalization of the presented method for a complete movement trajectory is possible. It is also interesting to notice the constancy of the existing error along the trajectory : there seems to be no important variation of the positioning error during the movement. We could therefore say that trajectories, as postures, could be calculated and then replayed with a good enough confidence.

\section{CONCLUSion}

Although exoskeletons kinematics is aimed at reproducing the human kinematics, there are unavoidable mismatches between the two chains. Therefore, directly mapping the human joint configuration to the robot joint configuration does not allow the exoskeleton to properly reproduce a recorded human movement. To overcome this problem, it is first necessary to guarantee the existence of a compatible configuration of the exoskeleton for a given limb posture, which is done, in this paper by the use of passive DoF fixations.

Secondly, inverse kinematics can be used. Thanks to the proposed method, based on generic robotic tools, postures kinematically compatible are computed for an exoskeleton with 2 interaction points with an acceptable error.

It is expected that this kind of tools and methods are becoming necessary for rehabilitation purposes. For example, with hemiparetic patients, a movement, or a coordination pattern, can be extracted from measurements of the healthy 

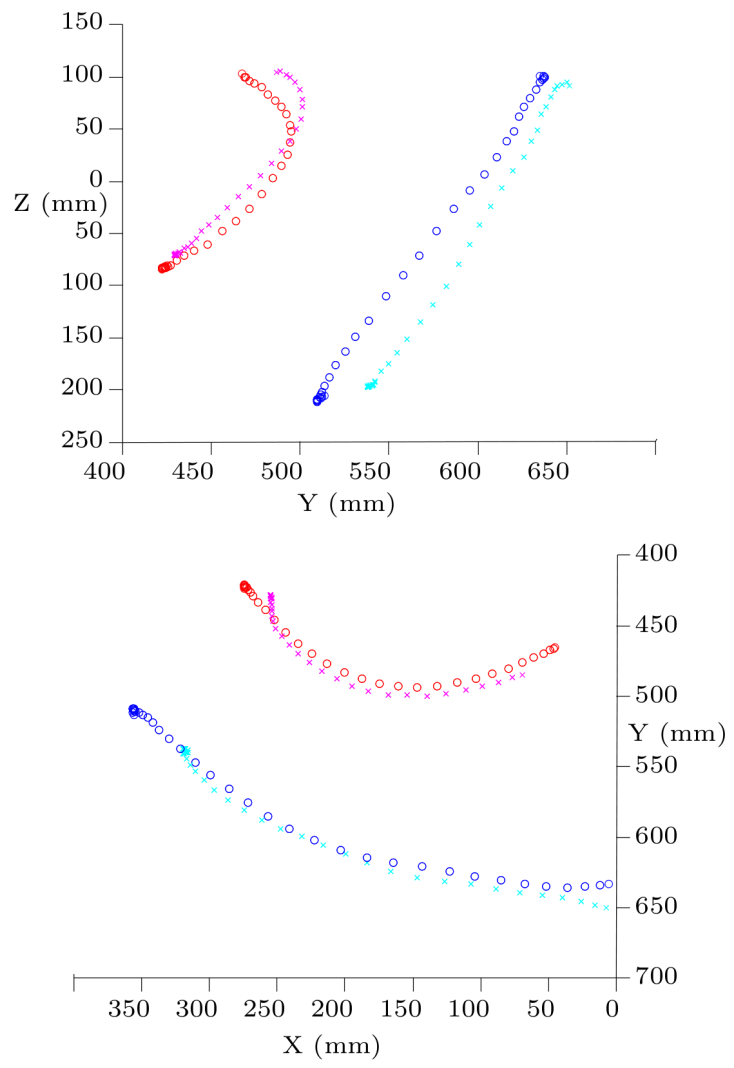

Fig. 8. Trajectories of the arm without (purple cross) and with robot (red circles). Trajectories of the forearm without (cyan cross) and with robot (blue circles).

limb in order to be used as a reference for the impaired limb. Only approaches similar to the one presented here will then guarantee a good reproduction of the recorded movement while avoiding the application by the robot of hyperstatic constraints on patient limb.

\section{ACKNOWLEDGMENTS}

This work was supported in part by the A.N.R. (Agence Nationale de la Recherche) within the project BRAHMA (BioRobotics for Assisting Human Manipulation) PSIROB 2006.

\section{REFERENCES}

[1] A.B. Zoss, H. Kazerooni, and A. Chu. Biomechanical design of the berkeley lower extremity exoskeleton (bleex). Mechatronics, IEEE/ASME Transactions on, 11(2):128 -138, april 2006.

[2] J.C. Perry, J. Rosen, and S. Burns. Exoskeletons for gait assistance and training of the motor-impaired. Mechatronics, IEEE/ASME Transactions on, Aug 2007.

[3] A. Frisoli, F. Rocchi, S. Marcheschi, A. Dettori, F. Salsedo, and M. Bergamasco. A new Force-Feedback arm exoskeleton for haptic interaction in virtual environments. In Proceedings of the First Joint Eurohaptics Conference and Symposium on Haptic Interfaces for Virtual Environment and Teleoperator Systems, pages 195-201. IEEE Computer Society, 2005.

[4] H. Vallery, E.H.F. van Asseldonk, M. Buss, and H. van der Kooij. Reference trajectory generation for rehabilitation robots: Complementary limb motion estimation. Neural Systems and Rehabilitation Engineering, IEEE Transactions on, 17(1):23 -30, feb. 2009.
[5] Alberto Montagner, Antonio Frisoli, Luigi Borelli, Caterina Procopio, Massimo Bergamasco, Maria C. Carboncini, and Bruno Rossi. A pilot clinical study on robotic assisted rehabilitation in VR with an arm exoskeleton device. In Virtual Rehabilitation, 2007, pages 57-64, 2007.

[6] Matja Mihelj, [1], Tobias [1] Nef, and Robert [1] Riener. A nove paradigm for patient-cooperative control of upper-limb rehabilitation robots. Advanced Robotics, 21:843-867, August 2007.

[7] G. Wu, F. C. van der Helm, H. E. Veeger, M. Makhsous, P. Van Roy, C. Anglin, J. Nagels, A. R. Karduna, K. McQuade, X. Wang, F. W. Werner, and B. Buchholz. Isb recommendation on definitions of joint coordinate systems of various joints for the reporting of human joint motion-part ii: shoulder, elbow, wrist and hand. Journal of Biomechanics, 35:543-548, May 2005.

[8] E V Biryukova, A Roby-Brami, A A Frolov, and M Mokhtari. Kinematics of human arm reconstructed from spatial tracking system recordings. Journal of Biomechanics, 33(8):985-995, August 2000 PMID: 10828329.

[9] J Leboucher, M Lempereur, S Brochard, and O Remy-Neris. Evaluation of joint coherence using fusionned data: Application to the gleno-humeral joint. In XXII Congress of the International Society of Biomechanics, 2009.

[10] S. J. Ball. Novel robotic mechanisms for upper-limb rehabilitation and assessment. $\mathrm{PhD}$ thesis, 2008

[11] R. Vertechy, A. Frisoli, A. Dettori, M. Solazzi, and M. Bergamasco. Development of a new exoskeleton for upper limb rehabilitation. pages $188-193$, jun. 2009.

[12] A.H.A Stienen, E.E.G. Hekman, F.C.T. van der Helm, and H. van der Kooij. Self-aligning exoskeleton axes through decoupling of joint rotations and translations. In Robotics, IEEE Transactions on, volume 25, June 2009.

[13] N. Jarrasse and G. Morel. On the kinematic design of exoskeletons and their fixations with a human member. In Proceedings of Robotics. Science and Systems, Zaragoza, Spain, June 2010.

[14] Fayet M. Mecanismes multi-boucles. Mechanism and Machine Theory, 30(2):201 - 252, 1995.

[15] P. Garrec, J.P. Friconneau, Y. Measson, and Y. Perrot. Able, an innovative transparent exoskeleton for the upper-limb. Intelligent Robots and Systems, 2008. IROS 2008. IEEE/RSJ International Conference on, pages 1483-1488, Sept. 2008

[16] Garrec. P. French patent: Transmission vis, ecrou et cable attache a la vis - fr0101630, 2000 (eur 01938347.0-2421 and us 10/296,740 (screw and nut transmission and cable). 2000. 